\title{
Homocysteine and Malondialdeyde (MDA) Levels Associated with the Occurrence of Cardiovascular Disease (CVD) in Chronic Renal Failure (CRF) in Malaysia
}

\author{
Shahin Arab \\ Department of Pathology, Chemical Pathology Unit, Faculty of Medicine and Health Science \\ University Putra Malaysia, 43400 UPM Serdang, Serdang Selangor, Malaysia \\ Tel: 60-17-226-5648Ｅ-mail: shahinarab1@gmail.com \\ Huzwah Khazaai (Corresponding author) \\ Department of Biomedical Science, Faculty of Medicine and Health Science, University Putra Malaysia \\ 43400 UPM Serdang, Serdang Selangor, Malaysia
}

Tel: 603-8947-2436 E-mail: huzwah@medic.upm.edu.my

Zarida Hambali

Department of Pathology, Chemical Pathology Unit, Faculty of Medicine and Health Science

University Putra Malaysia, 43400 UPM Serdang, Serdang Selangor, Malaysia

E-mail: zarida@mohe. gov.my

Zalinah Ahmad

Department of Pathology, Chemical Pathology Unit, Faculty of Medicine and Health Science

University Putra Malaysia, 43400 UPM Serdang, Serdang Selangor, Malaysia

E-mail: zalinah@medic.upm.edu.my

Received: July 30, 2010 Accepted: August 17, 2010 doi:10.5539/gjhs.v3n1p119

\begin{abstract}
Introduction: Atherosclerosis and following cardiovascular disease (CVD) are known as important reasons of the increased morbidity and mortality observed in patients with chronic renal failure (CRF). The association of serum malondialdehyde (MDA), homocysteine as well as other cardiovascular risk factors in relation to existence and cause of CVD were investigated.

Methods: In these study 66 CRF patients without dialysis and 107 patients receiving dialysis were recruited and further stratified into group with CVD and without CVD as case groups. Those without renal failure and CVD were assigned as control group $(\mathrm{n}=33)$.

Results: The patients with CRF showed a significant increase in plasma levels of MDA, homocysteine and C-reactive protein (CRP) compared to control. The positive association were observed between homocysteine, creatinine and MDA (all $p<0.01$ ) and another positive association were between CRP and age, creatinine and MDA (all $p<0.05)$. Analysis of association risk factors showed that only age, CRP and lipid profile had significant association with CVD events.

Conclusion: The results demonstrated elevation in plasma values of MDA, homocysteine and CRP in patients with CRF, with or without CVD. However, these modifications may be lead to atherosclerosis and consequence CVD event. These parameters may be important with respect to the high morbidity and mortality of CVD found in patients with CRF.
\end{abstract}

Keywords: Chronic Renal Failure (CRF), CVD, Hyperhomocysteinemia, Oxidative Stress 


\section{Introduction}

Atherosclerosis and CVD play an important role as a major source of morbidity and mortality in CRF patients. In addition, recent studies suggest that, cardiovascular injury started during early development of CRF complications (Nagane, 2009). Although traditional Framingham risk factors such as hypertension, dyslipidemia and diabetes may account for a large part of the excessive burden of CVD in CRF patients, non-traditional risk factors such as inflammation, oxidative stress and hyperhomocysteinemia may also contribute to the outcome of CVD in CRF patients (Stenvinkel et al., 2003).

Several epidemiological studies have reported that elevated total plasma homocysteine is a risk factor for cardiovascular disease. Homocysteine contains a reactive sulfhydryl group, and undergoes oxidation to disulfide at physiological $\mathrm{pH}$ in the presence of $\mathrm{O}_{2}$ (Mallamaci et al., 2002). It has been suggested that this pro-oxidant activity of homocysteine is also responsible for the oxidation of LDL cholesterol and damaging of vascular cells and tissues (Landry et al., 2006).

Hyperhomocysteinemia and lipid abnormalities are commonly found in patients with CRF; both are recognized as risk factors for atherosclerosis (Dom et al., 2003). Inflammation plays a central role in the pathogenesis of atherosclerosis in CRF patients. C-reactive protein (CRP) level was found to be elevated in kidney failure and may be related to cardiovascular complications (Menon et al., 2003; Wong et al., 2007). Due to the high mortality and morbidity rate in CRF patients caused by CVD, it is vital to find way which could reduce the death toll in these patients.

The current study was conducted to determine the role of non-traditional risk factors in the development of cardiovascular disease in dialysis and pre-dialysis patients. In this regard, biomarker of oxidative stress, homocysteine, CRP and lipid profile were measured.

\section{Material and Method}

\subsection{Subjects}

Individuals with CRF, with and without CVD from Serdang Hospital Selangor, Malaysia were recruited for this study. Patients with CRF were selected base on glomerular filtration rate (eGFR), CKD stage 1-4 (pre-dialysis) and CKD stage 5 (dialysis) were recruited (Levey et al., 2002). The selected population consisted of 85(49\%) male $88(51 \%)$ females, age between 18-80 years. Out of 173 patients, 66 were CRF patients with GFR (glomerular filtration rate) more than $15 \mathrm{ml} / \mathrm{min} / 1.73 \mathrm{~m}^{2}$ pre-dialysis whereas 107 patients with GFR less than 15 $\mathrm{ml} / \mathrm{min} / 1.73 \mathrm{~m}^{2}$ receiving dialysis and were clinically stable and free of active infections or autoimmune diseases. Those receiving immunosuppressive treatment and non-steroidal anti-inflammatory drugs were excluded from the study. Seventy six CRF patients had clinical symptoms or a previous history of cardiovascular disease (history of coronary heart disease or myocardial infarction, chronic ischemic heart disease, atherosclerosis, ischemic heart disease and congestive heart failure). The control group consist of 33 healthy subjects between the age of 18-80 years, who had no history of CRF or CVD. The study was approved by the Ethic Committee of Faculty of Medicine and Health Science, University Putra Malaysia (UPM). The informed consent was obtained from the subjects before participating in this study.

\subsection{Blood sampling and laboratory measurements}

Blood samples from CRF patients and controls were taken under fasting condition into tubes containing EDTA. The plasma were prepared conventionally, aliquoted and stored at $-80^{\circ} \mathrm{C}$ until the analysis were carried out.

\subsection{Routine parameters}

Lipid profile (HDL-C, LDL-C, total Cholesterol, triglyceride) were determined by enzymatic method using Roche/Hitachi analyzer (Roche Diagnostic, USA).

C-reactive protein (CRP) was measured by Selectra Flexor/XL autoanalyzer. This assay was based on the principle of particle-enhanced immunological agglutination or immunoturbidimetric assay. Following agglutination, this was measured turbidimetrically. Assay principle homocysteine was an enzyme immunoassay for the determination of total homocysteine in blood with an ELISA kit (AXIS, EIA pakage Insert, UK). MDA was measured using the established thiobarbituric acid (TBARS) method. MDA level of the plasma was measured according to the Tukozkan et al. (2006). The standards of 1,1,3,3 tetraetoxypropane (TEP) were used for preparation of standard curve. The results were expressed as nmol $/ \mathrm{ml}$ plasma.

\subsection{Statistical analysis}

The results were presented as the means \pm SD for normal and median (range) for skewed distributed variables. The significant differences of normal distributed variables between 2 groups were assessed by t-test and analysis 
of variance (ANOVA), while variables with non-Gaussian distribution were compared by using nonparametric Mann-Whitney and Kruskal-Wallis tests, respectively. All p-values were two-tailed and differences were considered significant if $p$-values were $\leq 0.05$. The association between the dependent variable and independent variables was determined by the correlation test. Associations of risk factors for CVD outcome by using logistic regression analysis was performed between two groups of CRF, with and without CVD as the dependent variable. All anthropometric, physiological and biochemical variables were incorporated and finally association of several risk factors with CVD outcome in CRF patients were evaluated.

\section{Results}

\subsection{Descriptive and ANOVA statistic}

The prevalence of demographic and clinical characteristics observed variables in CRF patients and healthy control are summarized in Table 1. There were significant differences in mean of the age in CRF and dialysis with CVD compared with control group. Dialysis groups had higher duration of CRF compared to group pre-dialysis. All groups showed no significant difference in systolic blood pressure but group with CRF without CVD had significantly high level of diastolic blood pressure compared to other groups. There were significant different in the antihyperlipidaemic and hypoglycaemic drugs consumption. CRF group without CVD had significant highest consumption of antihyperlipidemic drug, as well as CRF group with CVD for hypoglycaemic drug compared to other groups. As shown in Table 2, MDA concentrations were significantly different between $\mathrm{CRF}$ and control group. Among those involved in the study, CRF group receiving dialysis with CVD had the highest mean of MDA value. The levels of serum $\mathrm{C}$ - reactive protein in different groups of CRF was significantly higher than control, with CRF receiving dialysis and having CVD has the highest compared to other groups. Homocysteine concentrations in different group of CRF were significantly higher than control and CRF group receiving dialysis had higher levels of homocysteine compared to other groups. Plasma total cholesterol was significantly higher in all CRF groups compared to control group. Plasma triglyceride was only different in CRF without CVD compared to control group. However, there were no significant changes in level of HDL and LDL in all CRF patients compared to control group.

\subsection{The Pearson correlation coefficients ( $r$ ) analysis}

The Pearson correlation coefficients (r) of homocysteine, CRP and MDA levels relative to other parameters were carried out. Plasma level of homocysteine was positively and significantly associated with creatinine and MDA levels with $(\mathrm{r}=0.279$, and $\mathrm{r}=0.276$, both $p<0.01)$ respectively. The positive and significant correlation was observed between CRP and age, creatinine, MDA and homocysteine levels with $(r=0.174, r=0.130, r=0.157$ and $\mathrm{r}=0.155$, all $p<0.05$ ) respectively and inversely, correlated with HDL. The correlation of MDA concentration was positively and significantly with age $(\mathrm{r}=0.248, p<0.01)$, homocysteine $(\mathrm{r}=0.276, p<0.01)$, and negatively with HDL $(\mathrm{r}=-0.162, p<0.05)$.

\subsection{Associations of Risk Factors for CVD outcome by using Logistic regression analysis}

Logistic regression analysis was performed between two groups' CRF without CVD and CRF with CVD as the dependent variables (Table 4). All anthropometric and biochemical variables were keyed in; however, only age $(\mathrm{OR}=1.003$, CI 1.000-1.005, $p<0.001)$, CRP $(\mathrm{OR}=1.010$, CI 1.000-1.003, $p<0.05)$ and lipid profile showed significant association between these variables and occurrence of CVD. These associations in age, CRP, cholesterol, LDL and triglyceride showed strong regression in adjusted odds ratio.

\subsection{Evaluation of longitudinal study end point of cardiovascular disease}

In longitudinal study, type of cardiovascular event (MI, death, stroke and revascularization) were examined among the CRF patients with complete follow-up, which data are available. At the end of one year observation, of 173 CRF patients, 14 patients $(8 \%)$ were died. Of these, seven patients of CRF with CVD receiving haemodialysis which account $4 \%$ of the sample number died. On the other hand, 2 CRF without CVD patients $(1.2 \%)$ receiving CAPD (continuous ambulatory peritoneal dialysis), 1 patient of pre-dialysis without CVD $(0.6 \%)$ and 4 patients in pre-dialysis with CVD (2.4\%) died within that one year period (Table 5). In one whole year follow up only 1 patient $(0.6 \%)$ belong to the haemodialysis and another one of pre-dialysis with CVD $(0.6 \%)$ had myocardial infarction (MI). Only one of haemodialysis with CVD patient had revascularization $(0.6 \%)$.

\section{Discussion}

The results of the present study indicated that CRF patients had significantly increased levels of homocysteine, CRP and MDA as non-traditional risk factors compared to control subjects which are in agreement with other reports (Nagane, 2009; Vaziri, 2004). 


\subsection{Socio-demographic and clinical characteristic of CRF patients}

The results in this study revealed that duration of CRF complication were much longer especially in CRF patient with dialysis and CVD than other groups. Similarly, patient in our hospital were much older in dialysis group with CVD. Logistic regression analysis also showed, there were significant relationship between age and prevalence of CVD in CRF patients. These results support the observations in other study of Brenda and co-workers (Brenda et al., 2007) that, renal insufficiency is a marker for elevated cardiovascular disease risk in community dwelling with elderly adults. The most interesting observation in the present study was the presence of direct correlation between CVD prevalence and aging-associated inflammation. This result agrees with Pawlak and co-worker studies (Pawlak et al., 2005).

\subsection{Biochemical parameters of CRF patients}

There are several observational studies examining the relationship between dyslipidemia and CVD outcome (Shoji et al., 2001). Logistic regression analysis showed that CRF patients with high levels of triglyceride, LDL and cholesterol had significant association with the occurrence of CVD. The results of present study agreed with other investigators revealed those classic risk factors of atherosclerosis, age, duration of CRF and elevated lipid profiles, contribute to the development of carotid atherosclerosis in patients with CRF (Shoji et al., 2001; Leskinen et al., 2004). Determination of MDA, as the product of the oxidation of polyunsaturated fatty acid was reported to be an appropriate indirect method that revealed oxidation status in CRF patients. The significantly elevated serum MDA concentration in CRF patients compared to controls reflects the increased formation of ROS and lipid peroxidation which is in accordance to Kim et al., (2009). Meanwhile, CRF patient underwent dialysis with CVD showed higher mean of MDA levels than other groups. Similarly to our observation, Prakash et al., (2010) also found dialysis patients were exposed to oxidant stress by the presence of free radical and had greater impairment compared to patients without dialysis. In recent years, the role of endothelial cell dysfunction and oxidative stress for development of CVD has been highlighted. The mechanisms involved free radical attacking on cell membrane polyunsaturated fatty acids results in formation of lipid peroxidation products such as MDA, which altered the function of endothelial cell and vascular smooth muscle (Sasikala et al., 2000). The present study demonstrated a significant correlation between lipid peroxidation and oxidative stress in CRF patients (Annuk et al.,2003). Hyperhomocysteinemia, abnormalities in lipid profile and lipid peroxidation are commonly found in patients with CRF which are recognized as risk factors for atherosclerosis (Dom et al., 2003). Endothelium dysfunction and oxidative stress may be responsible for a metabolic block distally in transsulfuration route or at the homocysteine remethylation site causing hyperhomocysteinemia (Guldener, 2006; Guldener, 2005). Hyperhomocysteinemia, oxidative stress and endothelial dysfunction may be organized, forming a flow of atherothrombotic processes in CRF patients (Negane, 2009). Our data revealed that all CRF groups had significantly high level of homocysteine than control group with the highest concentration observed in CRF patient receiving dialysis with CVD compared to other groups, suggest patients incline towards experiencing a CVD event in ESRD (end stage renal disease) patients (Mallamaci et al., 2002). Mallamaci and co-worker (2002) observed a clear association between high level of homocysteine and incident of cardiovascular mortality and atherothrombotic events in haemodialysis patients. In this regard, our study showed positive correlation between homocysteine, creatinine and MDA level, and was inversely correlated with HDL concentration. The pathologic mechanisms involved in atherosclerosis remain unclear but experimental data support a range of possibilities, including hyperhomocysteinemia, endothelial cell injury (Lim PS, 2001), enhanced LDL oxidation (Querfeld, 2001) increased thromboxane-mediated platelet aggregation (Mackman, 2008) and inhibition of the anticoagulant protein $\mathrm{C}$ and promotion of smooth muscle cell proliferation. Homocysteine interferes with the coagulation system and lead to prothrombotic effects. It is recognized 30-50\% of pre-dialysis and dialysis patients have activated inflammatory response (Stenvinkel et al., 2002). Our results showed that, CRF patients had significant high concentration of CRP compared with control groups. Our study showed significant relationship between CRP and increase risk of CVD in CRF patients. Oxidative stress may have association between endothelial dysfunction and chronic inflammation in CRF patients. Our data is in agreement with some other reports which have shown high CRP levels in CRF and dialysis patients predict a constant inflammatory state on follow up, that showed significant relationship between CRP and increase risk of CVD (Ortega et al., 2001; Wong et al., 2007). On the other hand, CRP is explained to play a role in multiple stages of atherosclerotic lesion development (Lacsan et al., 2004). The mechanisms involved in CRP seems to be a direct mediator of endothelial dysfunction and inflammatory cell employment via the up-regulation of expression of endothelial cell adhesion molecules, by rising the release of monocyte chemotactic protein (MCP-1)( Devaraj et al., 2004) and/ or endothelial derived contracting factors and by inhibiting the nitric oxide production (Jialal et al., 2006). CRP promotes the uptake of low density lipoproteins (LDL) by foam cells 
(Snyder et al., 2005), which contributes to fibrous plaque formation and inhibits the remediable mechanism of angiogenesis (Rattazzi et al., 2003).

\subsection{Evaluation of cohort study}

In our cohort at Serdang Hospital, Malaysia the results showed $78.5 \%$ of deaths were among the patient with CVD. The results presented in this study were in agreement with numerous evident that patients with CRF have a substantially increased risk of CVD (Panza, 2009). The non traditional risk factors in this study such as hyperhomocsytienemia, CRP, lipid peroxidation marker had strong association with the status of the CRF patients in our hospital which attribute the severity of the chronic renal failure of the patients. This finding may have important clinical consequences (Negane, 2009; Pawlak et al, 2006). It is becoming evident that appropriate management of CVD in patients with CRF and dialysis may play a significant role in lowering the incidence of CVD morbidity and mortality in this population. Data collected from this study is hope to get more attention by the clinician in taking a proper measures in reducing the death toll of CRF patient that leading to CVD.

\section{Conclusion}

In conclusion, the findings of this study indicated significant elevation in plasma MDA, CRP and homocysteine in CRF patients, with or without CVD. However, it appeared increased level of oxidative stress marker which was accompanied with hyperhomocysteinemia and lipid peroxidation. These factors may be important with respect to the high morbidity and mortality of CVD found in patients with CRF, probably through correlation with endothelial dysfunction. However, the current research is the first work in Malaysia, but prospective studies are needed to determine with certainty the degree of CRF and CVD associated with changes with respect to non-traditional risk factors.

\section{Acknowledgements}

The authors thank Assoc. Prof. Dr. Alinda Chiu and Dr. Yap Yee Guan for their support and contribution this study. I would like to express my appreciation to all Pathology Laboratory members for their help in experimental works and all haemodialysis unit members at Serdang Hospital. The authors would also like to disclose that there is no conflict of interest involved while this project was carried out. This project was supported by IRPA grant 03-02-04-0562.

\section{References}

Annuk, M., Zilmer, M., Fellstrom, B. (2003). Endothelium-dependent vasodilatation and oxidative stress in chronic renal failure: impact on cardiovascular disease. Kidney Int Suppl, 84, S50-53.

Bennett, RK.., Kattenhorn, M., Hons, A., Donald AVT, Oakley Z \& RGN Z. (2002). Does Oral Folic Acid, Lower Total Homocysteine Levels and Improve Endothelial Function in Children With Chronic Renal Failure? Circulation, 1805-1810.

Brenda, R., Hemmelgarn. \& Braden, J., Zhang, MJ, Tonelli, M., Klarenbach, S., Walsh, M \& Bruce, F. (2007). Association between Multidisciplinary Care and Survival for Elderly Patients with Chronic Kidney Disease. $J$ Am Soc Nephrol, 18, 993-999.

Devaraj, S., Pappanaicken, R.. Kumaresan \& Jialal I. (2004). Effect of C-reactive protein on chemokine expression in human aortic endothelial cells. Journal of Molecular and Cellular Cardiology, 36, 405-410.

Dumm, NTG., Giammona, AM \& Touceda, TA. (2003). Variations in the lipid profile of patients with chronic renal failure treated with pyridoxine. Bio Med Central, 2(7), 2-7.

Guldener, CV. (2005). Homocysteine and the kidney. Current Drug Metabolism, 6, 23-26.

Guldener, CV. (2006). Why homocysteine elevated in renal failure and what is can be expected from homocysteine lowering? Nephrology Dialysis Transplantation, 21, 1161-1166.

Herzig, KA., Purdie, DM., Chang, W., Brown, AM., Hawley, CM \& Campbell, SB. (2001). Is C-reactive protein is a useful predictor of outcome in peritoneal dialysis patients? Journal of American Society of Nephrology, 12, 814-821.

Jialal, I., Devaraj S \& Uma, Singh. (2006). C - reactive protein and the Vascular Endothelium Implications for Plaque Instability. Journal of the American College of Cardiology, 47(7), 1379-1381.

Kim, HJ., Moradi, H., Yuan, J., Norris, K \& Vaziri, ND. (2009). Renal mass reduction results in accumulation of lipids and dysregulation of lipid regulatory proteins in the remnant kidney. Am J Physiol Renal Physiol, 296(6), F1297-306. 
Kronenberg, F., Neyer, U., Lhotta, K.., Trenkwalder, E., Auinger, M., Pribasnig, A,, Meisl, T., Konig, P \& Dieplinger, H. (1999). The low molecular weight apo(a) phenotype is an independent predictor for coronary artery disease in haemodialysis patients: a prospective follow-up. J Am Soc Nephrol, 10, 1027-1036.

Lacsan, EJ \& Levint, NW. (2004). C-reactive protein and end-stage renal disease. Seminars in Dialysis, 17, 438-448.

Landry, DW. \& Oliver, AJ. (2006). Blood pressure instability during hemodialysis Blood pressure instability during hemodialysis. Kidney International, 69, 1710-1711.

Leskinen, Y., Lehtimäki, T., Loimaala, A., Huhtala, H., Salenius, JP., Oja, SS \& Saha, H. (2004). Homocysteine and carotid atherosclerosis in chronic renal failure--the confounding effect of renal function. Atherosclerosis, 175(2), 315-23.

Levey, AS \& Coresh, J. (2002). K/DOQI clinical practice guidelines forchronic kidney disease evaluation, classification, and stratification. Kidney Disease Outcome Quality Initiative. Am J Kidney Dis, 39(suppl), S1-S246.

Lim, PS., Hung, WR., Wei, YH. (2001). Polymorphism in methyl tetra hydrofolate reductase gene its impact of plasma homocysteine levels and carotid atherosclerosis in ESRD patients receiving haemodialysis. Nephron, 87, 249-56.

Mackman, N. (2008). Review Article Triggers, targets and treatments for thrombosis. Nature, 451, 914-918.

Mallamaci, F., Zoccali, C., Tripepi, G., Fermo, I., Benedetto, FA., Cataliotti, A., Malatino, LS., Bellanuova I \& Soldarini, A. (2002). Hyperhomocysteinemia predicts cardiovascular outcomes in hemodialysis patients. Kidney Int, 61, 609-614.

Menon, V., Wang, X., Greene \& T. (2003). Relationship between C-reactive protein, albumin, and cardiovascular disease in patients with chronic kidney disease. Am J Kidney Dis, 42, 44-52.

Nagane, NS. (2009). Oxidative stress, serum homocysteine and serum nitric oxide in different stages of chronic renal failure, Ind Medica, 20(1) (2009-01 - 2009-04).

Ortega, O., Rodriguez, I., Gallar, P., Carreno, A., Ortiz, M \& Espejo, B. (2002). Significance of high C-reactive protein levels in predialysis patients. Nephrology Dialysis Transplant, 17, 1105-1109.

Panza, V., Pighin, D., Lainez, V., Poller, J.R \& Maldonado S. (2009). Storage lipids and poteins of Euterpe edulis seeds. Bio cell, 33(2), 99-106.

Pawlak, K \& Pawlak, D., Michael. (2006). Inflammation but not oxidative stress is associated with $\beta$-chemokine levels and prevalence of cardiovascular disease in uraemic patients. Cytokine, 35, 258-262.

Pawlak, P., Pawlak, D., Mysliwiec, M. (2005). Cu/Zn superoxide dismutase plasma levels as a new useful clinical biomarker of oxidative stress in patients with end-stage renal disease. Clinical Biochemistry, 38, 700705.

Prakash,M., Phani, NM., Kavya, R., Supriya. M. (2010). Paraoxonase: Its antiatherogenic role in chronic renal failure. Indian Journal of Nephrology, 20 (1 ), 9-14.

Querfeld U. (2001). Under treatment of Cardiac risk factors in adolescents with renal failure. Peritoneal Dialysis International, Vol. 21: Supplement 3.

Rattazzi, M., Puato, M., Faggin, E., Bertipaglia, B., Grego, F \& Pauletto P. (2003). New markers of accelerated atherosclerosis in end-stage renal disease. Journal of Nephrology, 16, 11-20.

Sasikala, M., Sadasivudu, B., Subramanyam, C. (2000). A Putative Role for Calcineurin in Lymphopenia Associated with Chronic Renal Failure. Clinical biochemistry, 33 (8), 691-694.

Shoji,T., Ishimura, E., Inaba, M., Tabata, T \& Nishizawa, Y. (2001). Atherogenic lipoproteins in end-stage renal disease. Am J Kidney Dis, 38, S30-S33.

Snyder, S \& Pendergraph, B. (2005). Detection and Evaluation of Chronic Kidney Disease. American Family Physician, 72, 1722-1732.

Stenvinkel, P. (2002). Inflammation in end-stage renal disease: could it be treated. Nephrology Dialysis Transplantation, 17 (8), 33-38.

Stenvinkel, P., Pecoits-Filho, R \& Lindholm, B. (2003). Coronary Artery Disease in End-Stage Renal Disease: No Longer a Simple Plumbing Problem. J Am Soc Nephrol, 14, 1927-1939. 
Thambyrajah, J \& Townend, JN. (2000). Homocysteine and atherothrombosis mechanism for injury, Eur Heart J, 21, 967- 47.

Tukozkan, N., Erdamar, H \& Seven, I. (2004). Measurement of total Malondialdehyde in plasma and tissues by high-performance liquid chromatography and tiobarbituric acid assay. Firat Tip Dergisi, 11(2), 88-92.

Vaziri, ND. (2004). Oxidative stress in uremia: nature, mechanisms, and potential consequences. Semin Nephrol, 24(5), 469-73.

Wong, LY., Leung, RY., Ong, KL \& Cheung, BM. (2007). Plasma levels of fibrinogen and C-reactive protein are regulated to interleukin-6 gene-572C $>$ G polymorphism in subjects with and without hypertension. $J$ Hum Hypertens, 21, 875-882.

Table 1. Demographic and clinical characteristics of pre-dialysis and dialysis with and Without CVD versus the control

\begin{tabular}{|l|l|l|l|l|l|}
\hline & Control & \multicolumn{2}{l|}{ pre-dialysis } & \multicolumn{2}{l|}{ dialysis } \\
\hline & & with CVD & with CVD & with CVD & with CVD \\
\hline Number & 33 & 33 & 33 & 41 & 66 \\
\hline Age (years) & $47.5 \pm 16.0$ & $61.3 \pm 13.5^{\text {a }}$ & $52.6 \pm 16.0$ & $60.4 \pm 12.5^{\text {a }}$ & $50.3 \pm 13.6$ \\
\hline BMI $\left(\mathrm{kg} / \mathrm{m}^{2}\right)$ & $23.7 \pm 3.3^{\mathrm{Aa}}$ & $24.8 \pm 3.7^{\mathrm{Aa}}$ & $28.3 \pm 4.9$ & $23.6 \pm 3.9^{\mathrm{Aa}}$ & $24.4 \pm 3.4^{\mathrm{Aa}}$ \\
\hline Waist circum (cm) & $83.4 \pm 9.8$ & $89.2 \pm 9.2$ & $93.4 \pm 9.9^{\mathrm{a}}$ & $86.7 \pm 9.8$ & $86.4 \pm 10.1$ \\
\hline Duration of CRF & ----- & $37.3 \pm 12.5$ & $25.2 \pm 6.4$ & $60.2 \pm 17.8^{\mathrm{c}}$ & $65.3 \pm 20.4^{\mathrm{c}}$ \\
\hline Smoking, \%(n) & $12(4)$ & $15(5)$ & $24(8)$ & $26(11)$ & $12(7)$ \\
\hline Systolic BP(mmHg) & $126.3 \pm 16.2$ & $129.3 \pm 26.2$ & $147.6 \pm 26.3^{\mathrm{b}}$ & $147.3 \pm 25.1^{\mathrm{b}}$ & $140.0 \pm 24.3^{\mathrm{b}}$ \\
\hline Diastolic BP( mmHg) & $78.7 \pm 11.2$ & $70 \pm 14.1^{*}$ & $84.8 \pm 16.3$ & $75 \pm 11.1^{*}$ & $78.3 \pm 12.2$ \\
\hline Druges & & & & & \\
\hline Antilipidemic, \% (n) & ----- & $28(4)$ & $33(11)^{*}$ & $29(12)$ & $13.5(9)$ \\
\hline Hypoglycaemic, \% (n) & ----- & $70(23)^{* *}$ & $33(11)$ & $50(20)$ & $13.5(9)$ \\
\hline Antihypertension, \% (n) & ----- & $50(15)^{* * *}$ & $52(16)^{* * *}$ & $39(16)$ & $30(19)$ \\
\hline
\end{tabular}

Note: Data were presented using ANOVA post test (Tukey test) and t test, ${ }^{\mathrm{a}} p<0.05,{ }^{\mathrm{b}} p<0.001$ patients versus control and, ${ }^{\text {Aa }} p<0.05$ pre-dialysis without CVD versus other groups, ${ }^{*} p<0.05$ pre-dialysis $\&$ dialysis with CVD versus. Pre-dialysis without CVD, ${ }^{* *} p<0.05$ pre-dialysis with CVD versus other groups. ${ }^{* * *} p<0.05$ pre-dialysis groups versus dialysis groups. ${ }^{c} p<0.05$ pre-dialysis without CVD versus dialysis groups. 
Table 2. Biochemical parameters of pre-dialysis and dialysis, with or without CVD versus the control

\begin{tabular}{|l|c|c|c|c|l|}
\hline Variables & Control & \multicolumn{2}{|c|}{ Pre- dialysis } & \multicolumn{2}{c|}{ dialysis } \\
\hline & & with CVD & without CVD & with CVD & without CVD \\
\hline MDA $(\mathrm{nmol} / \mathrm{mL})$ & $3.19 \pm 0.90$ & $5.28 \pm 1.02^{\mathrm{b}, \mathrm{c}}$ & $5.81 \pm 1.40^{\mathrm{b}}$ & $6.11 \pm 1.71^{\mathrm{b}, \mathrm{c}}$ & $5.40 \pm 1.40^{\mathrm{b}}$ \\
\hline Homocysteine $(\mu \mathrm{mol} / \mathrm{L})$ & $7.20 \pm 3.22$ & $15.0 \pm 7.50^{\mathrm{a}}$ & $12.60 \pm 6.91^{\mathrm{d}}$ & $19.60 \pm 12.10^{\mathrm{b}, \mathrm{d}}$ & $18.40 \pm 9.30^{\mathrm{b}, \mathrm{d}}$ \\
\hline CRP $(\mathrm{mg} / \mathrm{L})$ & $14.67 \pm 3.51$ & $41.13 \pm 18.60^{\mathrm{b}, \mathrm{d}}$ & $29.03 \pm 8.20^{\mathrm{b}}$ & $38.98 \pm 24.00^{\mathrm{b}, \mathrm{d}}$ & $28.81 \pm 17.26^{\mathrm{b}}$ \\
\hline Cholesterol $(\mathrm{mmol} / \mathrm{L})$ & $4.40 \pm 0.51$ & $5.40 \pm 053^{\mathrm{b}}$ & $5.61 \pm 1.22^{\mathrm{b}}$ & $5.20 \pm 1.21^{\mathrm{a}}$ & $5.10 \pm 1.25^{\mathrm{a}}$ \\
\hline LDL-c $(\mathrm{mmol} / \mathrm{L})$ & $2.60 \pm 0.51$ & $2.90 \pm 0.80$ & $3.10 \pm 1.00$ & $3.00 \pm 0.91$ & $3.00 \pm 1.10$ \\
\hline Triglyceride $(\mathrm{mmol} / \mathrm{L})$ & $1.05 \pm 0.38$ & $2.32 \pm 0.92^{\mathrm{b}}$ & $2.02 \pm 1.04^{\mathrm{b}}$ & $2.23 \pm 1.56^{\mathrm{b}}$ & $2.02 \pm 1.04^{\mathrm{b}}$ \\
\hline HDL-c $(\mathrm{mmol} / \mathrm{L})$ & $1.30 \pm 0.31$ & $1.11 \pm 0.30$ & $1.10 \pm 0.40$ & $1.05 \pm 0.33$ & $1.10 \pm 0.40$ \\
\hline
\end{tabular}

Note: Data were presented using ANOVA post test (Tukey test). ${ }^{\mathrm{a}} p<0.05,{ }^{\mathrm{b}} p<0.001$ patients versus control and, ${ }^{\mathrm{c}} p<0.05$ dialysis with CVD versus pre-dialysis with CVD, ${ }^{\mathrm{d}} p<0.05$ dialysis and pre-dialysis with CVD versus without CVD groups.

Table 3. Pearson correlation coefficients (r) of Homocysteine, CRP and MDA levels relative to another parameter

\begin{tabular}{|l|l|l|l|}
\hline Variable & Correlation & coefficient (r) & \\
\hline & Homocysteine & CRP & MDA \\
\hline Age & 0.113 & $0.174^{\mathrm{a}}$ & $0.083^{\mathrm{b}}$ \\
\hline Creatinine & $0.279^{\mathrm{b}}$ & $0.130^{\mathrm{a}}$ & 0.087 \\
\hline CRP & 0.152 & ------ & 0.157 \\
\hline MDA & $0.276^{\mathrm{b}}$ & $0.157^{\mathrm{a}}$ & ----- \\
\hline Homocysteine & ------ & 0.152 & $0.276^{\mathrm{b}}$ \\
\hline Cholesterol & 0.097 & $0.155^{\mathrm{b}}$ & 0.058 \\
\hline LDL-c & 0.111 & $0.039^{2}$ & 0.012 \\
\hline HDL-c & -0.103 & $-0.279^{\mathrm{b}}$ & $-0.279^{\mathrm{a}}$ \\
\hline Triglyceride & 0.092 & $0.413^{\mathrm{b}}$ & 0.413 \\
\hline
\end{tabular}

Note: Bivariate correlation analysis was performed using SPSS software. Superscript letters a, b indicate that the correlation coefficient are statistically significant at the confidence levels of ${ }^{\mathrm{a}} p<0.05,{ }^{\mathrm{b}} p<0.01$, respectively.

Table 4. Logistic regression analysis

\begin{tabular}{|l|l|l|l|}
\hline Variables & OR(adjusted) $\operatorname{Exp(\boldsymbol {B})}$ & $\boldsymbol{p}$ & $\mathbf{9 5 \%} \mathbf{C l}$ \\
\hline Age & 1.003 & 0.003 & $1.000-1.005$ \\
\hline CRP & 1.010 & 0.050 & $1.000-1.003$ \\
\hline Cholesterol & 1.211 & 0.020 & $1.101-1.301$ \\
\hline Triglyceride & 1.595 & 0.040 & $1.012-2.514$ \\
\hline LDL-c & 2.418 & 0.050 & $1.000-5.875$ \\
\hline
\end{tabular}

Note: The odds ratio for continuous variables are presented as the standardized regression coefficients as the term Exp (ß); OR, odds ratio; $\mathrm{Cl}$, confidence interval. 
Table 5. Follow up end point of CVD in CRF patients

\begin{tabular}{|l|l|l|l|l|l|l|}
\hline \multirow{2}{*}{} & \multicolumn{2}{|c|}{ Pre-dialysis } & \multicolumn{2}{c|}{ dialysis CAPD } & \multicolumn{2}{c|}{ Haemodialysis } \\
\cline { 2 - 8 } & With & $\begin{array}{l}\text { without } \\
\text { CVD }\end{array}$ & $\begin{array}{l}\text { With } \\
\text { CVD }\end{array}$ & $\begin{array}{l}\text { without } \\
\text { CVD }\end{array}$ & $\begin{array}{l}\text { WVD } \\
\text { CVD }\end{array}$ & $\begin{array}{l}\text { WVD } \\
\text { CVD }\end{array}$ \\
\hline MI (n) & 1 & --- & --- & --- & 1 & --- \\
\hline Stroke(n) & --- & --- & --- & --- & --- & --- \\
\hline Revascularization(n) & --- & --- & --- & --- & 1 & --- \\
\hline Dead (n) & 4 & 1 & --- & 2 & 7 & ---- \\
& & & & & & \\
\hline
\end{tabular}

Note: Evaluation of end point of CVD in CRF patients with and without CVD in one whole year. Abbreviation: CAPD (peritoneal dialysis). 